for

\title{
A New Azaferrocenophane with Azobenzene-containing Ligand. Remote control of Photoisomerization of the Azobenzene Group by Redox of Fe Center
}

\author{
Masaki Horie, Tatsuaki Sakano, Kohtaro Osakada*, and Hidenobu Nakao
}

Chemical Resources Laboratory, Tokyo Institute of Technology, 4259 Nagatsuta, Midori-ku, Yokohama, 226-8503, Japan

Experimental Section $\quad$ S1

$\begin{array}{lll}\text { Figure S1 } & \text { Flow electrolysis cell } & \text { S1 }\end{array}$

$\begin{array}{lll}\text { Figure S2 molecular structure of trans-1 } & \text { S3 }\end{array}$

Crystallographic Results of trans-1 $\quad$ S4

$\begin{array}{lll}\text { Figure S3-S5 first-order kinetics of } \mathbf{1} & \text { S10 }\end{array}$ 


\section{Experimental Section}

General Methods. $\quad \mathrm{RuCl}_{2}\left(\mathrm{PPh}_{3}\right) 3,{ }^{[1]} 1,1^{\prime}$-Ferrocenedimathanol, ${ }^{[2]} \mathrm{Pd} 2(\mathrm{dba}) 3^{[3]}$ and were prepared according to the literature. Preparation of the complexes and ferrocenophanes were carried out under nitrogen or argon using a standard Schlenk technique. Solvents were distilled from $\mathrm{CaH}_{2}$ and stored under argon. The IR spectra were obtained on a JASCO-IR810 spectrophotometer. The ${ }^{1} \mathrm{H}$ and ${ }^{13} \mathrm{C}$ NMR spectra were recorded on a JEOL EX-400 spectrometer at $25{ }^{\circ} \mathrm{C}$ unless otherwise stated. A YANACO MT-5 CHN Autocorder was used in the elemental analyses. Cyclic voltammetry was recorded in a $\mathrm{MeCN}$ solution of $0.10 \mathrm{M} \mathrm{Et}_{4} \mathrm{NBF}_{4}$ with an ALS Electrochemical Analyzer Model-600A. The potentials were referenced to $\mathrm{Ag}^{+} / \mathrm{Ag}$. Spectrochemical measurements were carried out by using a combination of a flow through electrolysis cell, ALS Electrochemical Analyzer Model-600A and EYELA peristaltic pump SMP-11, and JASCO V-530 UV/VIS spectrometer. Details of apparatus were shown in Figure S1. ${ }^{[4],[5]}$

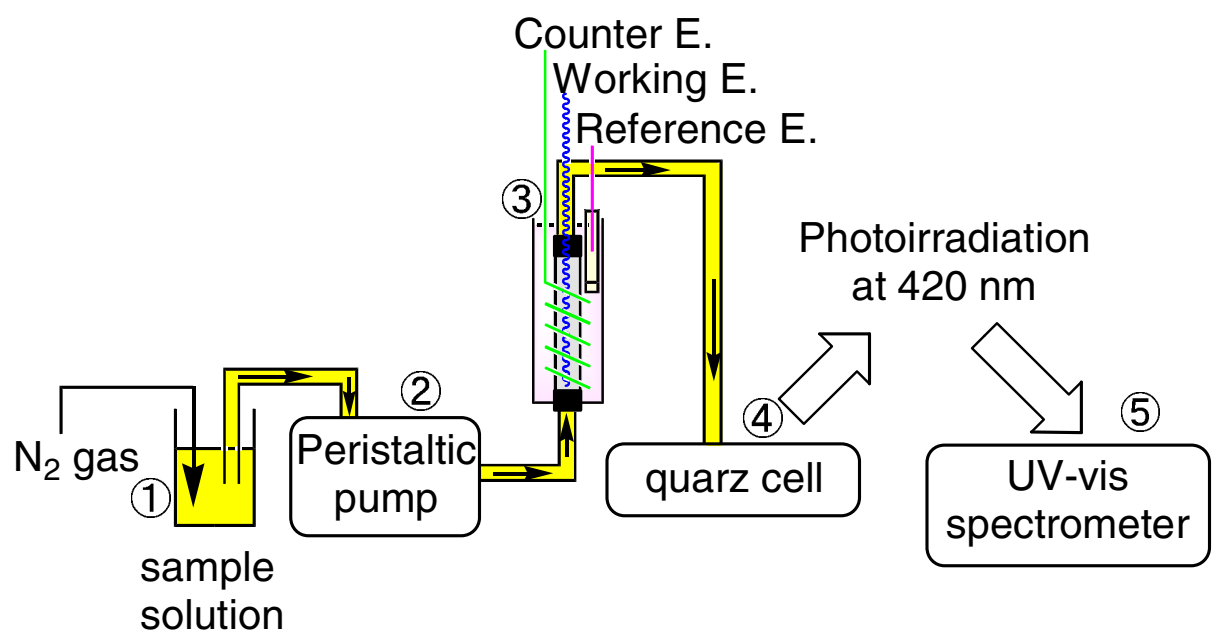

Figure S1 Schematic illustration of flow electrolysis system. 
Preparation of $\mathrm{N}$-(3-bromophenyl)-2-aza-[3]-ferrocenophane. To an NMP (5 mL) solution of $\mathrm{RuCl}_{2}\left(\mathrm{PPh}_{3}\right)_{3}(139 \mathrm{mg}, 0.15 \mathrm{mmol})$ was added 3-bromoaniline $(571 \mathrm{mg}, 3.3 \mathrm{mmol})$ and then $1,1^{\prime}$-ferrocenedimethanol $(817 \mathrm{mg}, 3.3 \mathrm{mmol})$ at room temperature under nitrogen atmosphere. The mixture was heated for $24 \mathrm{~h}$ at $180{ }^{\circ} \mathrm{C}$. After removal of the solvent under vacuum, the product was extracted with acetone. Column chromatography (silica gel; hexane: ethyl acetate $=1: 1$ ) of the extract gave a yellow solid which was recrystallized from a dichloromethane-methanol mixture to afford $\mathrm{N}$-(3-bromophenyl)-2-aza-[3]-ferrocenophane as yellow crystals (907 $\mathrm{mg}, 68 \%$ ). Anal. Calcd. for $\mathrm{C}_{18} \mathrm{H}_{16} \mathrm{FeNBr}$ : C, 56.58; H, 4.22; N, 3.67; Br, 20.91. Found for C, 56.82; H, 4.31; $\mathrm{N}, 3.65 .{ }^{1} \mathrm{H}$ NMR $\left(400 \mathrm{MHz}\right.$, in $\mathrm{CDCl}_{3}$ at $\left.25^{\circ} \mathrm{C}\right): \delta 7.09\left(\mathrm{~d}, 1 \mathrm{H}\right.$, ortho, $\left.\mathrm{BrC}_{6} \mathrm{H}_{4}, J=7 \mathrm{~Hz}\right), 7.08(\mathrm{dd}$, $1 \mathrm{H}$, meta, $\left.\mathrm{Br}_{6} \mathrm{H}_{4}, J=7 \mathrm{~Hz}\right), 6.85\left(\mathrm{~s}, 1 \mathrm{H}\right.$, ortho, $\left.\mathrm{Br}_{6} \mathrm{H}_{4}\right), 6.84\left(\mathrm{~d}, 1 \mathrm{H}\right.$, para, $\left.\mathrm{Br}_{6} \mathrm{H}_{4}, J=7 \mathrm{~Hz}\right)$, 4.18, $4.06(\mathrm{t}, 8 \mathrm{H}, \mathrm{Cp}, J=2 \mathrm{~Hz}), 3.84\left(\mathrm{~s}, 4 \mathrm{H}, \mathrm{Cp}-\mathrm{CH}_{2}-\mathrm{N}\right) .{ }^{13} \mathrm{C}\left\{{ }^{1} \mathrm{H}\right\} \mathrm{NMR}\left(100 \mathrm{MHz}\right.$, in $\mathrm{CDCl}_{3}$ at $25^{\circ} \mathrm{C}$ ): $\delta 150.4$ (meta, $\mathrm{Br} \mathrm{C}_{6} \mathrm{H}_{4}-\mathrm{N}$ ), 130.6 (meta, $\mathrm{Br}_{6} \mathrm{H}_{4}$ ), 123.7 (ipso, $\mathrm{Br}_{6} \mathrm{H}_{4}$ ), 119.8 (ortho, $\mathrm{Br}$ $\mathrm{C}_{6} \mathrm{H}_{4}$ ), 115.9 (ortho, $\mathrm{Br} \mathrm{C}_{6} \mathrm{H}_{4}-\mathrm{N}$ ), 111.7 (para, $\mathrm{Br} \mathrm{C}_{6} \mathrm{H}_{4}$ ), $83.8\left(\right.$ (Cp- $\mathrm{CH}_{2}-$ ), 69.9, 69.3 (Cp), 46.0 $\left(\mathrm{Cp}-\mathrm{CH}_{2}-\mathrm{N}\right)$.

Preparation of $N$-\{3-(phenylazo)phenyl\}aminophenyl-2-aza-[3]-ferrocenophane (trans-1). A mixture of $N$-(3-bromophenyl)-2-aza-[3]-ferrocenophane $(382 \mathrm{mg}, 1.0 \mathrm{mmol})$ and 4-phenylazodiphenylamine (273 mg, $1.0 \mathrm{mmol})$ was dissolved in toluene $(20 \mathrm{~mL}) . \quad \mathrm{NaO}-t$ - Bu $(145$ $\mathrm{mg}, 1.5 \mathrm{mmol}), \mathrm{Pd}_{2}(\mathrm{dba})_{3}(12 \mathrm{mg}, 0.013 \mathrm{mmol}), \mathrm{P}(t-\mathrm{Bu})_{3}(15 \mathrm{mg}, 0.075 \mathrm{mmol})$ were added to the solution. This solution was stirred at $100{ }^{\circ} \mathrm{C}$ for $40 \mathrm{~h}$ under argon. After cooling, reaction mixture was quenched by adding $\mathrm{NH}_{4} \mathrm{OH}$. The product was extracted by $\mathrm{CHCl}_{3}$ and purified by column chromatography (silica gel; hexane: $\mathrm{CHCl}_{3}=1: 1$ ) to give a red-orange solid. Recrystallization from a $\mathrm{CHCl}_{3} /$ hexane mixture gave 2 as red crystals $(471 \mathrm{mg}, 82 \%)$. Anal. Calcd. for $\mathrm{C}_{36} \mathrm{H}_{30} \mathrm{FeN}_{4}$ : C, 75.26; H, 5.26; N, 9.75. Found for C, 75.14; H, 5.13; N, 9.65. ${ }^{1} \mathrm{H}$ NMR (400 MHz, in $\mathrm{CDCl}_{3}$ at $25^{\circ} \mathrm{C}$ ): $\delta 7.87-6.55$ (aromatic region), 4.17, 4.07 (d, $8 \mathrm{H}, \mathrm{Cp}, J=2 \mathrm{~Hz}$ ), 3.81 (s, $\left.4 \mathrm{H}, \mathrm{Cp}-\mathrm{CH}_{2}-\mathrm{N}\right) .{ }^{13} \mathrm{C}\left\{{ }^{1} \mathrm{H}\right\}$ NMR $\left(100 \mathrm{MHz}\right.$, in $\mathrm{CDCl}_{3}$ at $\left.25^{\circ} \mathrm{C}\right): \delta 153.0,150.7,150.4,148.2$, 147.0, 130.4, 130.1, 129.4, 129.0, 125.4, 124.2, 123.9, 122.5, 121.3, 115.5, 111.1, 109.7 (aromatic

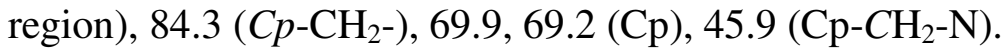

Kinetic Measurement. A toluene solution of trans $-1(0.03 \mathrm{mM})$ was oxidized by passing the flow electrolysis system in Figure S1. The solution in a quartz cell was irradiated at $420 \mathrm{~nm}$ using Xe lamp filtered by color glass filter for $30 \mathrm{~min}$ at $20^{\circ} \mathrm{C}$. The spectroscopic change was measured by increase of the absorption at $430 \mathrm{~nm}$. The results are shown in Figure 3 (b).

\section{References}

[1] P. S. Hallman, T. A. Stephenson, G. Wilkinson, Inorg. Synth. 1970, 12, 237.

[2] A. -S. Carlström, T. Frejd, J. Org. Chem. 1990, 55, 4175.

[3] T. Ukai, H. Kawazura, Y. Ishii, J. Organomet. Chem. 1974, 65, 253.

[4] H. Nakao, H. Hayashi, K. Okita, Anal. Sci. 2001, 17, 545.

[5] T. Sakano, M. Horie, K. Osakada, H. Nakao, Bull. Chem. Soc. Jpn. 2001, 74, 2059. 


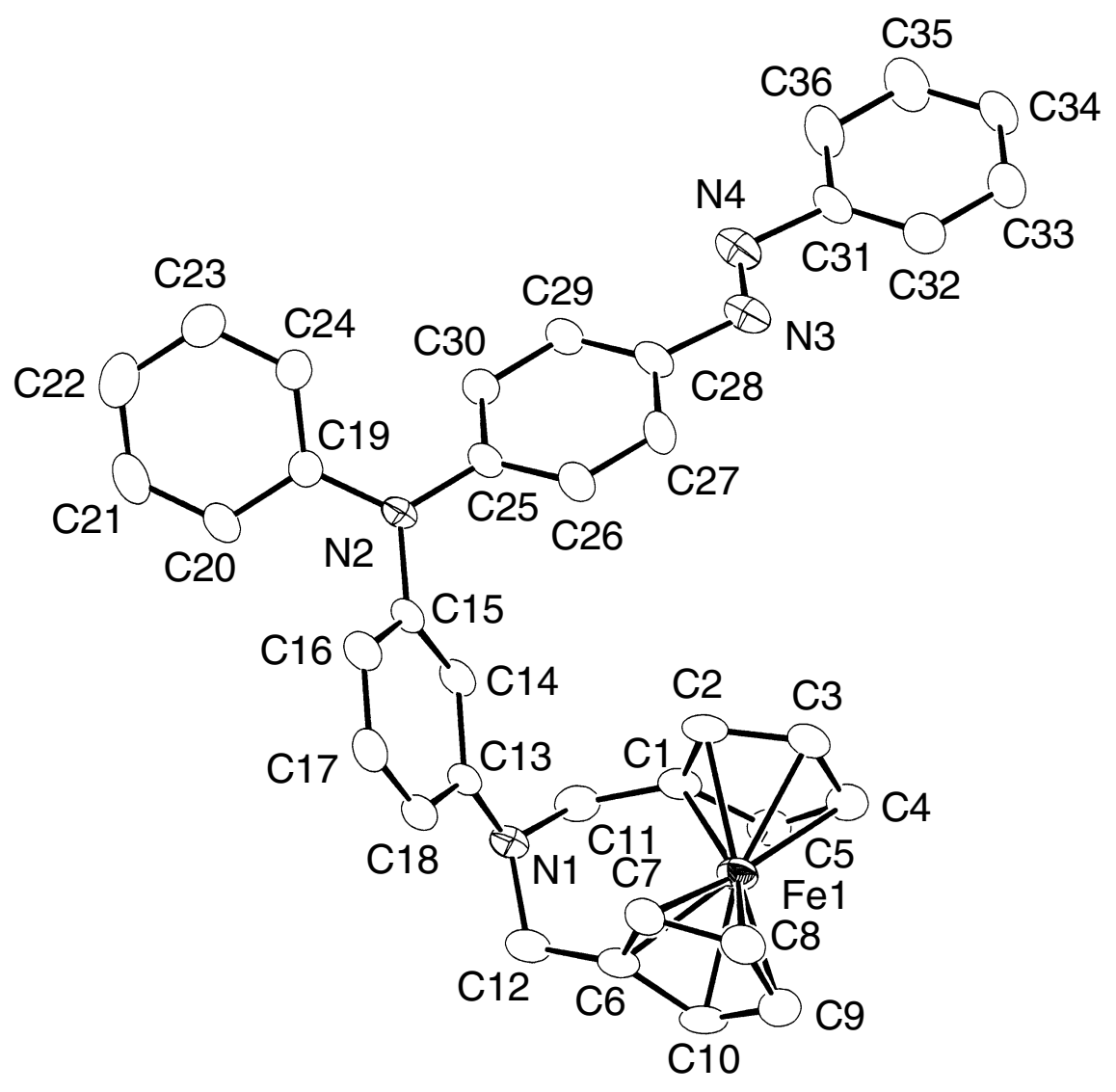

Figure S2 Horie, M.; Sakano, T.; Osakada, K.; Nakao, H. 


\section{Crystallographic Results of trans-1}

\section{EXPERIMENTAL DETAILS}

Empirical Formula

Formula Weight

Crystal Color, Habit

Crystal Dimensions

Crystal System

Lattice Type

No. of Reflections Used for Unit

Cell Determination (2 $\theta$ range)

Omega Scan Peak Width

at Half-height

Lattice Parameters

Space Group

$Z$ value

$\mathrm{D}_{\text {calc }}$

F000

$\mu(\mathrm{MoK} \alpha)$

Diffractometer

Radiation

Attenuator

Take-off Angle

Detector Aperture

Crystal to Detector Distance

Voltage, Current

Temperature

Scan Type

Scan Rate

Scan Width

$2 \theta_{\max }$

No. of Reflections Measured

Corrections
A. Crystal Data

$$
\mathrm{C}_{36} \mathrm{H}_{30} \mathrm{~N}_{4} \mathrm{Fe}
$$

574.51

red, prismatic

0.40 X $0.50 \times 0.70 \mathrm{~mm}$

triclinic

Primitive

$20\left(7.7-12.4^{0}\right)$

0.270

$\mathrm{a}=10.362(2) \AA$

$\mathrm{b}=18.639(4) \AA$

$\mathrm{c}=7.399(2) \AA$

$\alpha=97.97(2) \AA$

$\beta=102.67(2) \AA$

$\gamma=86.58(2) \AA$

$\mathrm{V}=1380.1(5) \AA^{3}$

P-1 (\#2)

2

$1.382 \mathrm{~g} / \mathrm{cm}^{3}$

600.00

$5.79 \mathrm{~cm}^{-1}$

B. Intensity Measurements

Rigaku AFC5R

$\operatorname{MoK} \alpha(\lambda=0.71069 \AA)$

graphite monochromated

$\mathrm{Zr}$ foil $($ factors $=1.00,3.51,11.52,42.17)$

$6.0^{\circ}$

$9.0 \mathrm{~mm}$ horizontal

$13.0 \mathrm{~mm}$ vertical

$258 \mathrm{~mm}$

$0 \mathrm{kV}, 0 \mathrm{~mA}$

$23.0^{\circ} \mathrm{C}$

$\omega-2 \theta$

$16.0^{\circ} / \mathrm{min}$ (in $\omega$ ) (up to 3 scans)

$(1.63+0.30 \tan \theta)^{\mathrm{O}}$

$55.0^{\circ}$

Total: 5457

Unique: $5105\left(\mathrm{R}_{\mathrm{int}}=0.051\right)$

Lorentz-polarization 
Structure Solution

Refinement

Function Minimized

Least Squares Weights

p-factor

Anomalous Dispersion

No. Observations (I>3.00 $\sigma(\mathrm{I}))$

No. Variables

Reflection/Parameter Ratio

Residuals: R; Rw

Goodness of Fit Indicator

Max Shift/Error in Final Cycle

Maximum peak in Final Diff. Map

Minimum peak in Final Diff. Map
Absorption

(trans. factors: 0.9169 - 1.0000)

C. Structure Solution and Refinement

Patterson Methods (DIRDIF92 PATTY)

Full-matrix least-squares on $\mathrm{F}$

$\Sigma \mathrm{w}(|\mathrm{Fo}|-|\mathrm{Fc}|)^{2}$

$1 / \sigma^{2}(\mathrm{Fo})=4 \mathrm{Fo}^{2} / \sigma^{2}\left(\mathrm{Fo}^{2}\right)$

0.0033

All non-hydrogen atoms

2381

370

6.44

$0.047 ; 0.033$

1.65

0.00

$0.00 \mathrm{e}^{-} / \AA^{3}$

$0.00 \mathrm{e}^{-} / \AA^{3}$

Table 1. Atomic coordinates and $\mathrm{B}_{\mathrm{iso}} / \mathrm{B}_{\mathrm{eq}}$

$\begin{array}{lcccl}\text { atom } & \mathrm{x} & \mathrm{y} & \mathrm{z} & \mathrm{B} \text { eq } \\ \mathrm{Fe}(1) & 0.04999(6) & 0.34085(4) & -0.04221(8) & 3.52(2) \\ \mathrm{N}(1) & -0.0670(3) & 0.2042(2) & 0.0942(4) & 3.42(9) \\ \mathrm{N}(2) & 0.2860(3) & 0.0796(2) & 0.4928(4) & 3.56(9) \\ \mathrm{N}(3) & 0.5365(3) & 0.3292(2) & 0.9047(5) & 4.7(1) \\ \mathrm{N}(4) & 0.5472(4) & 0.3356(2) & 1.0745(5) & 5.1(1) \\ \mathrm{C}(1) & 0.0331(4) & 0.3243(2) & 0.2143(5) & 3.4(1) \\ \mathrm{C}(2) & 0.1683(4) & 0.3124(2) & 0.1958(5) & 3.7(1) \\ \mathrm{C}(3) & 0.2115(4) & 0.3782(3) & 0.1549(5) & 4.5(1) \\ \mathrm{C}(4) & 0.1049(5) & 0.4303(2) & 0.1487(6) & 4.4(1) \\ \mathrm{C}(5) & -0.0029(5) & 0.3978(3) & 0.1877(5) & 4.1(1) \\ \mathrm{C}(6) & -0.0714(5) & 0.2626(2) & -0.1915(5) & 3.6(1) \\ \mathrm{C}(7) & 0.0588(4) & 0.2508(2) & -0.2293(5) & 3.6(1) \\ \mathrm{C}(8) & 0.0902(4) & 0.3124(3) & -0.3013(5) & 4.2(1) \\ \mathrm{C}(9) & -0.0178(5) & 0.3629(3) & -0.3116(6) & 4.6(1) \\ \mathrm{C}(10) & -0.1172(4) & 0.3324(3) & -0.2465(5) & 4.2(1) \\ \mathrm{C}(11) & -0.0602(4) & 0.2678(2) & 0.2345(5) & 3.8(1) \\ \mathrm{C}(12) & -0.1409(4) & 0.2176(2) & -0.0917(5) & 4.1(1) \\ \mathrm{C}(13) & 0.0313(4) & 0.1504(2) & 0.1136(5) & 3.1(1) \\ \mathrm{C}(14) & 0.1145(4) & 0.1427(2) & 0.2882(5) & 3.0(1) \\ \mathrm{C}(15) & 0.2069(4) & 0.0865(2) & 0.3095(5) & 3.2(1) \\ \mathrm{C}(16) & 0.2223(4) & 0.0352(2) & 0.1602(6) & 3.4(1) \\ \mathrm{C}(17) & 0.1413(4) & 0.0425(2) & -0.0100(5) & 3.6(1) \\ \mathrm{C}(18) & 0.0498(4) & 0.0982(2) & -0.0345(5) & 3.5(1) \\ \mathrm{C}(19) & 0.2921(4) & 0.0132(2) & 0.5629(5) & 3.2(1)\end{array}$




$\begin{array}{lllll}\mathrm{C}(20) & 0.1844(4) & -0.0320(2) & 0.5123(5) & 3.8(1) \\ \mathrm{C}(21) & 0.1917(5) & -0.0967(3) & 0.5823(6) & 4.9(1) \\ \mathrm{C}(22) & 0.3018(6) & -0.1187(3) & 0.7054(7) & 5.8(2) \\ \mathrm{C}(23) & 0.4091(5) & -0.0728(3) & 0.7569(6) & 5.1(1) \\ \mathrm{C}(24) & 0.4050(4) & -0.0097(2) & 0.6837(6) & 4.1(1) \\ \mathrm{C}(25) & 0.3506(4) & 0.1415(2) & 0.6014(6) & 3.3(1) \\ \mathrm{C}(26) & 0.4003(4) & 0.1924(2) & 0.5167(5) & 3.9(1) \\ \mathrm{C}(27) & 0.4605(4) & 0.2525(2) & 0.6205(6) & 4.2(1) \\ \mathrm{C}(28) & 0.4735(4) & 0.2632(2) & 0.8127(6) & 3.7(1) \\ \mathrm{C}(29) & 0.4252(4) & 0.2131(2) & 0.8986(5) & 4.0(1) \\ \mathrm{C}(30) & 0.3640(4) & 0.1527(2) & 0.7941(5) & 3.5(1) \\ \mathrm{C}(31) & 0.6062(4) & 0.4035(2) & 1.1706(6) & 4.2(1) \\ \mathrm{C}(32) & 0.6422(4) & 0.4576(3) & 1.0845(6) & 4.6(1) \\ \mathrm{C}(33) & 0.6937(5) & 0.5196(2) & 1.1957(6) & 4.9(1) \\ \mathrm{C}(34) & 0.7075(5) & 0.5274(2) & 1.3840(6) & 5.2(1) \\ \mathrm{C}(35) & 0.6719(6) & 0.4728(3) & 1.4660(7) & 7.6(2) \\ \mathrm{C}(36) & 0.6199(5) & 0.4114(3) & 1.3594(7) & 6.9(2)\end{array}$

$\mathrm{B}_{\mathrm{eq}}=8 / 3 \pi^{2}\left(\mathrm{U}_{11}\left(\mathrm{aa}^{*}\right)^{2}+\mathrm{U}_{22}\left(\mathrm{bb}^{*}\right)^{2}+\mathrm{U}_{33}(\mathrm{cc} *)^{2}+2 \mathrm{U}_{12}\left(\mathrm{aa}^{*} \mathrm{bb}\right) \cos \gamma+2 \mathrm{U}_{13}\left(\mathrm{aa}^{*} \mathrm{cc} *\right) \cos \beta+\right.$ $\left.2 \mathrm{U}_{23}(\mathrm{bb} * \mathrm{cc} *) \cos \alpha\right)$

Table 2. Anisotropic Displacement Parameters

$\begin{array}{lllllll}\text { atom } & \mathrm{U} 11 & \mathrm{U}_{22} & \mathrm{U} 33 & \mathrm{U} 12 & \mathrm{U} 13 & \mathrm{U} 23 \\ \mathrm{Fe}(1) & 0.0480(4) & 0.0483(4) & 0.0311(3) & -0.0004(3) & -0.0000(3) & -0.0028(3) \\ \mathrm{N}(1) & 0.041(2) & 0.050(2) & 0.035(2) & -0.006(2) & 0.001(2) & -0.000(2) \\ \mathrm{N}(2) & 0.043(2) & 0.042(2) & 0.042(2) & -0.009(2) & -0.008(2) & 0.001(2) \\ \mathrm{N}(3) & 0.047(3) & 0.067(3) & 0.055(3) & -0.008(2) & -0.001(2) & -0.004(2) \\ \mathrm{N}(4) & 0.057(3) & 0.069(3) & 0.061(3) & -0.015(2) & -0.002(2) & -0.004(2) \\ \mathrm{C}(1) & 0.046(3) & 0.055(3) & 0.024(2) & 0.002(3) & 0.002(2) & -0.004(2) \\ \mathrm{C}(2) & 0.043(3) & 0.062(4) & 0.029(2) & 0.004(3) & -0.004(2) & -0.002(2) \\ \mathrm{C}(3) & 0.059(4) & 0.065(4) & 0.039(3) & -0.018(3) & -0.008(2) & 0.002(2) \\ \mathrm{C}(4) & 0.074(4) & 0.045(3) & 0.038(3) & 0.000(3) & -0.004(3) & -0.006(2) \\ \mathrm{C}(5) & 0.064(4) & 0.047(3) & 0.037(3) & 0.008(3) & 0.001(3) & -0.006(2) \\ \mathrm{C}(6) & 0.054(3) & 0.053(3) & 0.023(2) & -0.004(3) & -0.006(2) & -0.004(2) \\ \mathrm{C}(7) & 0.054(3) & 0.044(3) & 0.032(2) & -0.003(3) & 0.006(2) & -0.009(2) \\ \mathrm{C}(8) & 0.060(4) & 0.063(4) & 0.033(2) & -0.006(3) & 0.011(2) & -0.004(2) \\ \mathrm{C}(9) & 0.076(4) & 0.058(4) & 0.036(3) & -0.008(3) & 0.001(3) & 0.006(2) \\ \mathrm{C}(10) & 0.055(3) & 0.065(4) & 0.028(2) & 0.006(3) & -0.010(2) & -0.001(2) \\ \mathrm{C}(11) & 0.041(3) & 0.058(3) & 0.041(3) & 0.012(3) & 0.008(2) & 0.003(2) \\ \mathrm{C}(12) & 0.044(3) & 0.062(3) & 0.044(3) & 0.001(3) & -0.001(2) & 0.001(2) \\ \mathrm{C}(13) & 0.034(3) & 0.043(3) & 0.038(2) & -0.012(2) & 0.000(2) & 0.003(2) \\ \mathrm{C}(14) & 0.040(3) & 0.041(3) & 0.032(2) & -0.009(2) & 0.009(2) & -0.010(2) \\ \mathrm{C}(15) & 0.039(3) & 0.041(3) & 0.039(2) & -0.015(2) & 0.001(2) & -0.000(2) \\ \mathrm{C}(16) & 0.038(3) & 0.046(3) & 0.046(3) & -0.006(2) & 0.012(2) & -0.004(2) \\ \mathrm{C}(17) & 0.062(3) & 0.041(3) & 0.036(3) & -0.014(3) & 0.019(2) & -0.011(2)\end{array}$




$\begin{array}{llllllr}\mathrm{C}(18) & 0.047(3) & 0.047(3) & 0.036(2) & -0.013(3) & 0.002(2) & -0.000(2) \\ \mathrm{C}(19) & 0.046(3) & 0.035(3) & 0.040(3) & -0.003(2) & 0.009(2) & 0.000(2) \\ \mathrm{C}(20) & 0.051(3) & 0.051(3) & 0.042(3) & -0.017(3) & 0.008(2) & -0.004(2) \\ \mathrm{C}(21) & 0.074(4) & 0.060(4) & 0.055(3) & -0.031(3) & 0.024(3) & -0.011(3) \\ \mathrm{C}(22) & 0.103(5) & 0.051(4) & 0.074(4) & -0.006(4) & 0.033(4) & 0.013(3) \\ \mathrm{C}(23) & 0.059(4) & 0.058(4) & 0.077(4) & 0.009(3) & 0.009(3) & 0.021(3) \\ \mathrm{C}(24) & 0.049(3) & 0.041(3) & 0.063(3) & -0.003(3) & 0.006(3) & 0.011(2) \\ \mathrm{C}(25) & 0.033(3) & 0.040(3) & 0.047(3) & -0.006(2) & 0.001(2) & -0.002(2) \\ \mathrm{C}(26) & 0.056(3) & 0.051(3) & 0.041(3) & -0.017(3) & 0.008(2) & -0.003(2) \\ \mathrm{C}(27) & 0.055(3) & 0.045(3) & 0.061(3) & -0.020(3) & 0.011(3) & 0.006(2) \\ \mathrm{C}(28) & 0.033(3) & 0.045(3) & 0.055(3) & -0.004(2) & -0.002(2) & -0.007(2) \\ \mathrm{C}(29) & 0.054(3) & 0.050(3) & 0.040(3) & -0.012(3) & -0.002(2) & -0.002(2) \\ \mathrm{C}(30) & 0.043(3) & 0.050(3) & 0.040(3) & -0.008(2) & 0.006(2) & 0.007(2) \\ \mathrm{C}(31) & 0.047(3) & 0.047(3) & 0.055(3) & -0.012(3) & -0.003(3) & -0.010(3) \\ \mathrm{C}(32) & 0.062(4) & 0.057(3) & 0.051(3) & -0.006(3) & 0.005(3) & 0.001(3) \\ \mathrm{C}(33) & 0.070(4) & 0.052(3) & 0.063(3) & -0.017(3) & 0.009(3) & 0.002(3) \\ \mathrm{C}(34) & 0.080(4) & 0.048(3) & 0.055(3) & -0.022(3) & -0.006(3) & -0.012(3) \\ \mathrm{C}(35) & 0.147(6) & 0.088(5) & 0.048(3) & -0.055(4) & 0.002(3) & -0.005(3) \\ \mathrm{C}(36) & 0.127(5) & 0.075(4) & 0.060(4) & -0.054(4) & 0.004(4) & 0.004(3)\end{array}$

The general temperature factor expression:

$\exp \left(-2 \pi^{2}\left(a^{*} \mathrm{U}_{11} \mathrm{~h}^{2}+\mathrm{b}^{* 2} \mathrm{U}_{22} \mathrm{k}^{2}+\mathrm{c}^{* 2} \mathrm{U}_{33} \mathrm{l}^{2}+2 \mathrm{a} * \mathrm{~b} * \mathrm{U}_{12} \mathrm{hk}+2 \mathrm{a} * \mathrm{c} * \mathrm{U}_{13} \mathrm{hl}+2 \mathrm{~b} * \mathrm{c}^{*} \mathrm{U}_{23} \mathrm{kl}\right)\right)$

Table 3. Bond Lengths $(\AA)$

$\begin{array}{llllll}\text { atom } & \text { atom } & \text { distance } & \text { atom } & \text { atom } & \text { distance } \\ \mathrm{Fe}(1) & \mathrm{C}(1) & 2.011(5) & \mathrm{Fe}(1) & \mathrm{C}(2) & 2.032(5) \\ \mathrm{Fe}(1) & \mathrm{C}(3) & 2.050(5) & \mathrm{Fe}(1) & \mathrm{C}(4) & 2.051(5) \\ \mathrm{Fe}(1) & \mathrm{C}(5) & 2.042(5) & \mathrm{Fe}(1) & \mathrm{C}(6) & 2.023(5) \\ \mathrm{Fe}(1) & \mathrm{C}(7) & 2.034(5) & \mathrm{Fe}(1) & \mathrm{C}(8) & 2.044(5) \\ \mathrm{Fe}(1) & \mathrm{C}(9) & 2.055(5) & \mathrm{Fe}(1) & \mathrm{C}(10) & 2.034(5) \\ \mathrm{N}(1) & \mathrm{C}(11) & 1.458(6) & \mathrm{N}(1) & \mathrm{C}(12) & 1.467(6) \\ \mathrm{N}(1) & \mathrm{C}(13) & 1.384(6) & \mathrm{N}(2) & \mathrm{C}(15) & 1.441(6) \\ \mathrm{N}(2) & \mathrm{C}(19) & 1.401(6) & \mathrm{N}(2) & \mathrm{C}(25) & 1.424(6) \\ \mathrm{N}(3) & \mathrm{N}(4) & 1.227(5) & \mathrm{N}(3) & \mathrm{C}(28) & 1.441(6) \\ \mathrm{N}(4) & \mathrm{C}(31) & 1.463(7) & \mathrm{C}(1) & \mathrm{C}(2) & 1.440(7) \\ \mathrm{C}(1) & \mathrm{C}(5) & 1.429(7) & \mathrm{C}(1) & \mathrm{C}(11) & 1.512(7) \\ \mathrm{C}(2) & \mathrm{C}(3) & 1.425(7) & \mathrm{C}(3) & \mathrm{C}(4) & 1.426(7) \\ \mathrm{C}(4) & \mathrm{C}(5) & 1.406(7) & \mathrm{C}(6) & \mathrm{C}(7) & 1.438(7) \\ \mathrm{C}(6) & \mathrm{C}(10) & 1.442(7) & \mathrm{C}(6) & \mathrm{C}(12) & 1.497(7) \\ \mathrm{C}(7) & \mathrm{C}(8) & 1.416(7) & \mathrm{C}(8) & \mathrm{C}(9) & 1.413(7) \\ \mathrm{C}(9) & \mathrm{C}(10) & 1.410(7) & \mathrm{C}(13) & \mathrm{C}(14) & 1.409(6) \\ \mathrm{C}(13) & \mathrm{C}(18) & 1.401(6) & \mathrm{C}(14) & \mathrm{C}(15) & 1.379(6) \\ \mathrm{C}(15) & \mathrm{C}(16) & 1.388(6) & \mathrm{C}(16) & \mathrm{C}(17) & 1.372(7) \\ \mathrm{C}(17) & \mathrm{C}(18) & 1.367(7) & \mathrm{C}(19) & \mathrm{C}(20) & 1.392(7) \\ \mathrm{C}(19) & \mathrm{C}(24) & 1.393(7) & \mathrm{C}(20) & \mathrm{C}(21) & 1.368(7)\end{array}$




$\begin{array}{llllll}\mathrm{C}(21) & \mathrm{C}(22) & 1.377(8) & \mathrm{C}(22) & \mathrm{C}(23) & 1.396(8) \\ \mathrm{C}(23) & \mathrm{C}(24) & 1.360(7) & \mathrm{C}(25) & \mathrm{C}(26) & 1.389(7) \\ \mathrm{C}(25) & \mathrm{C}(30) & 1.391(6) & \mathrm{C}(26) & \mathrm{C}(27) & 1.366(6) \\ \mathrm{C}(27) & \mathrm{C}(28) & 1.387(7) & \mathrm{C}(28) & \mathrm{C}(29) & 1.373(7) \\ \mathrm{C}(29) & \mathrm{C}(30) & 1.378(6) & \mathrm{C}(31) & \mathrm{C}(32) & 1.376(7) \\ \mathrm{C}(31) & \mathrm{C}(36) & 1.362(7) & \mathrm{C}(32) & \mathrm{C}(33) & 1.386(7) \\ \mathrm{C}(33) & \mathrm{C}(34) & 1.357(7) & \mathrm{C}(34) & \mathrm{C}(35) & 1.362(8) \\ \mathrm{C}(35) & \mathrm{C}(36) & 1.364(8) & & & \end{array}$

Table 4. Bond Angles( $\left(^{\circ}\right)$

\begin{tabular}{|c|c|c|c|c|c|c|c|}
\hline atom & atom & atom & angle & atom & atom & atom & angle \\
\hline $\mathrm{C}(1)$ & $\mathrm{Fe}(1)$ & $\mathrm{C}(2)$ & $41.7(2)$ & $\mathrm{C}(1)$ & $\mathrm{Fe}(1)$ & $\mathrm{C}(3)$ & $69.3(2)$ \\
\hline $\mathrm{C}(1)$ & $\mathrm{Fe}(1)$ & $C(4)$ & $69.0(2)$ & $\mathrm{C}(1)$ & $\mathrm{Fe}(1)$ & $C(5)$ & $41.3(2)$ \\
\hline $\mathrm{C}(1)$ & $\mathrm{Fe}(1)$ & $C(6)$ & $98.0(2)$ & $\mathrm{C}(1)$ & $\mathrm{Fe}(1)$ & $C(7)$ & $116.4(2)$ \\
\hline $\mathrm{C}(1)$ & $\mathrm{Fe}(1)$ & $\mathrm{C}(8)$ & $155.6(2)$ & $\mathrm{C}(1)$ & $\mathrm{Fe}(1)$ & $\mathrm{C}(9)$ & $155.5(2)$ \\
\hline $\mathrm{C}(1)$ & $\mathrm{Fe}(1)$ & $C(10)$ & $116.8(2)$ & $C(2)$ & $\mathrm{Fe}(1)$ & $\mathrm{C}(3)$ & $40.9(2)$ \\
\hline $\mathrm{C}(2)$ & $\mathrm{Fe}(1)$ & $\mathrm{C}(4)$ & $68.9(2)$ & $\mathrm{C}(2)$ & $\mathrm{Fe}(1)$ & $\mathrm{C}(5)$ & $69.2(2)$ \\
\hline $\mathrm{C}(2)$ & $\mathrm{Fe}(1)$ & $C(6)$ & $114.7(2)$ & $\mathrm{C}(2)$ & $\mathrm{Fe}(1)$ & $C(7)$ & $102.7(2)$ \\
\hline $\mathrm{C}(2)$ & $\mathrm{Fe}(1)$ & $\mathrm{C}(8)$ & $123.6(2)$ & $\mathrm{C}(2)$ & $\mathrm{Fe}(1)$ & $\mathrm{C}(9)$ & $162.6(2)$ \\
\hline $\mathrm{C}(2)$ & $\mathrm{Fe}(1)$ & $C(10)$ & $152.4(3)$ & $\mathrm{C}(3)$ & $\mathrm{Fe}(1)$ & $\mathrm{C}(4)$ & $40.7(2)$ \\
\hline $\mathrm{C}(3)$ & $\mathrm{Fe}(1)$ & $C(5)$ & $68.3(2)$ & $\mathrm{C}(3)$ & $\mathrm{Fe}(1)$ & $C(6)$ & $153.8(2)$ \\
\hline $\mathrm{C}(3)$ & $\mathrm{Fe}(1)$ & $C(7)$ & $122.5(2)$ & $\mathrm{C}(3)$ & $\mathrm{Fe}(1)$ & $\mathrm{C}(8)$ & $113.0(2)$ \\
\hline $\mathrm{C}(3)$ & $\mathrm{Fe}(1)$ & $\mathrm{C}(9)$ & $130.2(3)$ & $\mathrm{C}(3)$ & $\mathrm{Fe}(1)$ & $\mathrm{C}(10)$ & $164.6(3)$ \\
\hline $\mathrm{C}(4)$ & $\mathrm{Fe}(1)$ & $C(5)$ & $40.2(2)$ & $C(4)$ & $\mathrm{Fe}(1)$ & $\mathrm{C}(6)$ & $156.8(3)$ \\
\hline $\mathrm{C}(4)$ & $\mathrm{Fe}(1)$ & $C(7)$ & $161.5(2)$ & $\mathrm{C}(4)$ & $\mathrm{Fe}(1)$ & $\mathrm{C}(8)$ & $129.6(2)$ \\
\hline $\mathrm{C}(4)$ & $\mathrm{Fe}(1)$ & $C(9)$ & $114.4(2)$ & $\mathrm{C}(4)$ & $\mathrm{Fe}(1)$ & $\mathrm{C}(10)$ & $126.0(2)$ \\
\hline $\mathrm{C}(5)$ & $\mathrm{Fe}(1)$ & $C(6)$ & $117.7(2)$ & $C(5)$ & $\mathrm{Fe}(1)$ & $\mathrm{C}(7)$ & $154.0(2)$ \\
\hline $\mathrm{C}(5)$ & $\mathrm{Fe}(1)$ & $\mathrm{C}(8)$ & $163.1(2)$ & $C(5)$ & $\mathrm{Fe}(1)$ & $\mathrm{C}(9)$ & $125.1(2)$ \\
\hline $\mathrm{C}(5)$ & $\mathrm{Fe}(1)$ & $C(10)$ & $106.2(2)$ & $\mathrm{C}(6)$ & $\mathrm{Fe}(1)$ & $C(7)$ & $41.5(2)$ \\
\hline$C(6)$ & $\mathrm{Fe}(1)$ & $\mathrm{C}(8)$ & $69.2(2)$ & $\mathrm{C}(6)$ & $\mathrm{Fe}(1)$ & $\mathrm{C}(9)$ & $69.5(2)$ \\
\hline$C(6)$ & $\mathrm{Fe}(1)$ & $C(10)$ & $41.6(2)$ & $\mathrm{C}(7)$ & $\mathrm{Fe}(1)$ & $\mathrm{C}(8)$ & $40.6(2)$ \\
\hline $\mathrm{C}(7)$ & $\mathrm{Fe}(1)$ & $C(9)$ & $68.7(2)$ & $C(7)$ & $\mathrm{Fe}(1)$ & $C(10)$ & $69.0(2)$ \\
\hline $\mathrm{C}(8)$ & $\mathrm{Fe}(1)$ & $C(9)$ & $40.3(2)$ & $\mathrm{C}(8)$ & $\mathrm{Fe}(1)$ & $\mathrm{C}(10)$ & $67.9(2)$ \\
\hline $\mathrm{C}(9)$ & $\mathrm{Fe}(1)$ & $C(10)$ & $40.3(2)$ & $\mathrm{C}(11)$ & $\mathrm{N}(1)$ & $\mathrm{C}(12)$ & $113.4(4)$ \\
\hline$C(11)$ & $\mathrm{N}(1)$ & $\mathrm{C}(13)$ & $120.2(4)$ & $\mathrm{C}(12)$ & $\mathrm{N}(1)$ & $\mathrm{C}(13)$ & $120.5(4)$ \\
\hline$C(15)$ & $\mathrm{N}(2)$ & $C(19)$ & $119.1(4)$ & $C(15)$ & $\mathrm{N}(2)$ & $C(25)$ & $119.1(4)$ \\
\hline$C(19)$ & $\mathrm{N}(2)$ & $\mathrm{C}(25)$ & $121.7(4)$ & $\mathrm{N}(4)$ & $\mathrm{N}(3)$ & $\mathrm{C}(28)$ & $112.1(5)$ \\
\hline $\mathrm{N}(3)$ & $\mathrm{N}(4)$ & $\mathrm{C}(31)$ & $113.2(5)$ & $\mathrm{Fe}(1)$ & $\mathrm{C}(1)$ & $\mathrm{C}(2)$ & $69.9(3)$ \\
\hline $\mathrm{Fe}(1)$ & $\mathrm{C}(1)$ & $C(5)$ & $70.5(3)$ & $\mathrm{Fe}(1)$ & $\mathrm{C}(1)$ & $\mathrm{C}(11)$ & $119.5(3)$ \\
\hline $\mathrm{C}(2)$ & $\mathrm{C}(1)$ & $C(5)$ & $107.5(5)$ & $C(2)$ & $\mathrm{C}(1)$ & $\mathrm{C}(11)$ & $126.8(5)$ \\
\hline $\mathrm{C}(5)$ & $\mathrm{C}(1)$ & $\mathrm{C}(11)$ & $125.3(5)$ & $\mathrm{Fe}(1)$ & $\mathrm{C}(2)$ & $\mathrm{C}(1)$ & $68.4(3)$ \\
\hline $\mathrm{Fe}(1)$ & $\mathrm{C}(2)$ & $\mathrm{C}(3)$ & $70.3(3)$ & $\mathrm{C}(1)$ & $\mathrm{C}(2)$ & $\mathrm{C}(3)$ & $107.4(5)$ \\
\hline $\mathrm{Fe}(1)$ & $\mathrm{C}(3)$ & $\mathrm{C}(2)$ & $68.9(3)$ & $\mathrm{Fe}(1)$ & $\mathrm{C}(3)$ & $\mathrm{C}(4)$ & $69.7(3)$ \\
\hline $\mathrm{C}(2)$ & $\mathrm{C}(3)$ & $\mathrm{C}(4)$ & $108.2(5)$ & $C(2)$ & $\mathrm{C}(3)$ & $\mathrm{H}(2)$ & 125.2 \\
\hline $\mathrm{Fe}(1)$ & $\mathrm{C}(4)$ & $\mathrm{C}(3)$ & $69.6(3)$ & $\mathrm{Fe}(1)$ & $\mathrm{C}(4)$ & $\mathrm{C}(5)$ & $69.6(3)$ \\
\hline
\end{tabular}




\begin{tabular}{|c|c|c|c|c|c|c|c|}
\hline $\mathrm{C}(3)$ & $C(4)$ & $C(5)$ & $108.3(5)$ & $\mathrm{Fe}(1)$ & $C(5)$ & $\mathrm{C}(1)$ & $68.2(3)$ \\
\hline $\mathrm{Fe}(1)$ & $C(5)$ & $\mathrm{C}(4)$ & $70.3(3)$ & $\mathrm{C}(1)$ & $C(5)$ & $\mathrm{C}(4)$ & $108.6(5)$ \\
\hline $\mathrm{Fe}(1)$ & $C(6)$ & $C(7)$ & $69.7(3)$ & $\mathrm{Fe}(1)$ & $C(6)$ & $\mathrm{C}(10)$ & $69.6(3)$ \\
\hline $\mathrm{Fe}(1)$ & $C(6)$ & $\mathrm{C}(12)$ & $119.4(3)$ & $C(7)$ & $C(6)$ & $C(10)$ & $106.2(5)$ \\
\hline$C(7)$ & $C(6)$ & $\mathrm{C}(12)$ & $128.0(5)$ & $\mathrm{C}(10)$ & $C(6)$ & $\mathrm{C}(12)$ & $125.4(6)$ \\
\hline $\mathrm{Fe}(1)$ & $C(7)$ & $\mathrm{C}(6)$ & $68.8(3)$ & $\mathrm{Fe}(1)$ & $C(7)$ & $\mathrm{C}(8)$ & $70.0(3)$ \\
\hline$C(6)$ & $C(7)$ & $\mathrm{C}(8)$ & $107.9(5)$ & $\mathrm{Fe}(1)$ & $\mathrm{C}(8)$ & $\mathrm{C}(7)$ & $69.3(3)$ \\
\hline $\mathrm{Fe}(1)$ & $\mathrm{C}(8)$ & $\mathrm{C}(9)$ & $70.3(3)$ & $C(7)$ & $\mathrm{C}(8)$ & $\mathrm{C}(9)$ & $109.2(5)$ \\
\hline $\mathrm{Fe}(1)$ & $\mathrm{C}(9)$ & $\mathrm{C}(8)$ & $69.4(3)$ & $\mathrm{Fe}(1)$ & $\mathrm{C}(9)$ & $C(10)$ & $69.0(3)$ \\
\hline $\mathrm{C}(8)$ & $\mathrm{C}(9)$ & $\mathrm{C}(10)$ & $107.5(5)$ & $\mathrm{Fe}(1)$ & $\mathrm{C}(10)$ & $\mathrm{C}(6)$ & $68.8(3)$ \\
\hline $\mathrm{Fe}(1)$ & $C(10)$ & $\mathrm{C}(9)$ & $70.7(3)$ & $C(6)$ & $C(10)$ & $\mathrm{C}(9)$ & $109.2(5)$ \\
\hline $\mathrm{N}(1)$ & $\mathrm{C}(11)$ & $\mathrm{C}(1)$ & $113.8(4)$ & $\mathrm{N}(1)$ & $\mathrm{C}(12)$ & $\mathrm{C}(6)$ & $115.0(4)$ \\
\hline $\mathrm{N}(1)$ & $\mathrm{C}(13)$ & $\mathrm{C}(14)$ & $121.3(5)$ & $\mathrm{N}(1)$ & $\mathrm{C}(13)$ & $\mathrm{C}(18)$ & $122.8(5)$ \\
\hline$C(14)$ & $\mathrm{C}(13)$ & $\mathrm{C}(18)$ & $115.9(5)$ & $\mathrm{C}(13)$ & $\mathrm{C}(14)$ & $C(15)$ & $121.1(5)$ \\
\hline $\mathrm{N}(2)$ & $C(15)$ & $C(14)$ & $118.8(5)$ & $\mathrm{N}(2)$ & $C(15)$ & $C(16)$ & $119.3(5)$ \\
\hline$C(14)$ & $C(15)$ & $C(16)$ & $121.8(5)$ & $\mathrm{C}(15)$ & $C(16)$ & $\mathrm{C}(17)$ & $117.0(5)$ \\
\hline$C(16)$ & $C(17)$ & $\mathrm{C}(18)$ & $122.2(5)$ & $\mathrm{C}(13)$ & $\mathrm{C}(18)$ & $\mathrm{C}(17)$ & $121.9(5)$ \\
\hline $\mathrm{N}(2)$ & $C(19)$ & $C(20)$ & $120.4(5)$ & $\mathrm{N}(2)$ & $C(19)$ & $\mathrm{C}(24)$ & $121.2(5)$ \\
\hline$C(20)$ & $C(19)$ & $\mathrm{C}(24)$ & $118.4(5)$ & $\mathrm{C}(19)$ & $\mathrm{C}(20)$ & $\mathrm{C}(21)$ & $119.7(5)$ \\
\hline $\mathrm{C}(20)$ & $\mathrm{C}(21)$ & $\mathrm{C}(22)$ & $122.3(6)$ & $\mathrm{C}(21)$ & $\mathrm{C}(22)$ & $\mathrm{C}(23)$ & $117.6(6)$ \\
\hline$C(22)$ & $\mathrm{C}(23)$ & $\mathrm{C}(24)$ & $120.8(6)$ & C(19) & $C(24)$ & $\mathrm{C}(23)$ & $121.1(5)$ \\
\hline $\mathrm{N}(2)$ & $C(25)$ & $C(26)$ & $120.6(5)$ & $\mathrm{N}(2)$ & $C(25)$ & $C(30)$ & $120.7(5)$ \\
\hline$C(26)$ & $C(25)$ & $C(30)$ & $118.7(5)$ & $\mathrm{C}(25)$ & $C(26)$ & $C(27)$ & $120.6(5)$ \\
\hline$C(26)$ & $C(27)$ & $\mathrm{C}(28)$ & $120.2(5)$ & $\mathrm{N}(3)$ & $\mathrm{C}(28)$ & $\mathrm{C}(27)$ & $114.5(6)$ \\
\hline $\mathrm{N}(3)$ & $\mathrm{C}(28)$ & $C(29)$ & $125.6(5)$ & $\mathrm{C}(27)$ & $\mathrm{C}(28)$ & $C(29)$ & $119.9(5)$ \\
\hline $\mathrm{C}(28)$ & $C(29)$ & $C(30)$ & $120.0(5)$ & $\mathrm{C}(25)$ & $C(30)$ & $C(29)$ & $120.5(5)$ \\
\hline $\mathrm{N}(4)$ & $C(31)$ & $C(32)$ & $125.1(6)$ & $\mathrm{N}(4)$ & $\mathrm{C}(31)$ & $C(36)$ & $114.0(6)$ \\
\hline$C(32)$ & $C(31)$ & $C(36)$ & $120.8(6)$ & $\mathrm{C}(31)$ & $C(32)$ & $C(33)$ & $117.8(5)$ \\
\hline$C(32)$ & $C(33)$ & $C(34)$ & $121.4(6)$ & $\mathrm{C}(33)$ & $C(34)$ & $C(35)$ & $119.5(6)$ \\
\hline $\mathrm{C}(34)$ & $C(35)$ & $C(36)$ & $120.4(6)$ & $\mathrm{C}(31)$ & $\mathrm{C}(36)$ & $C(35)$ & $120.1(6)$ \\
\hline
\end{tabular}



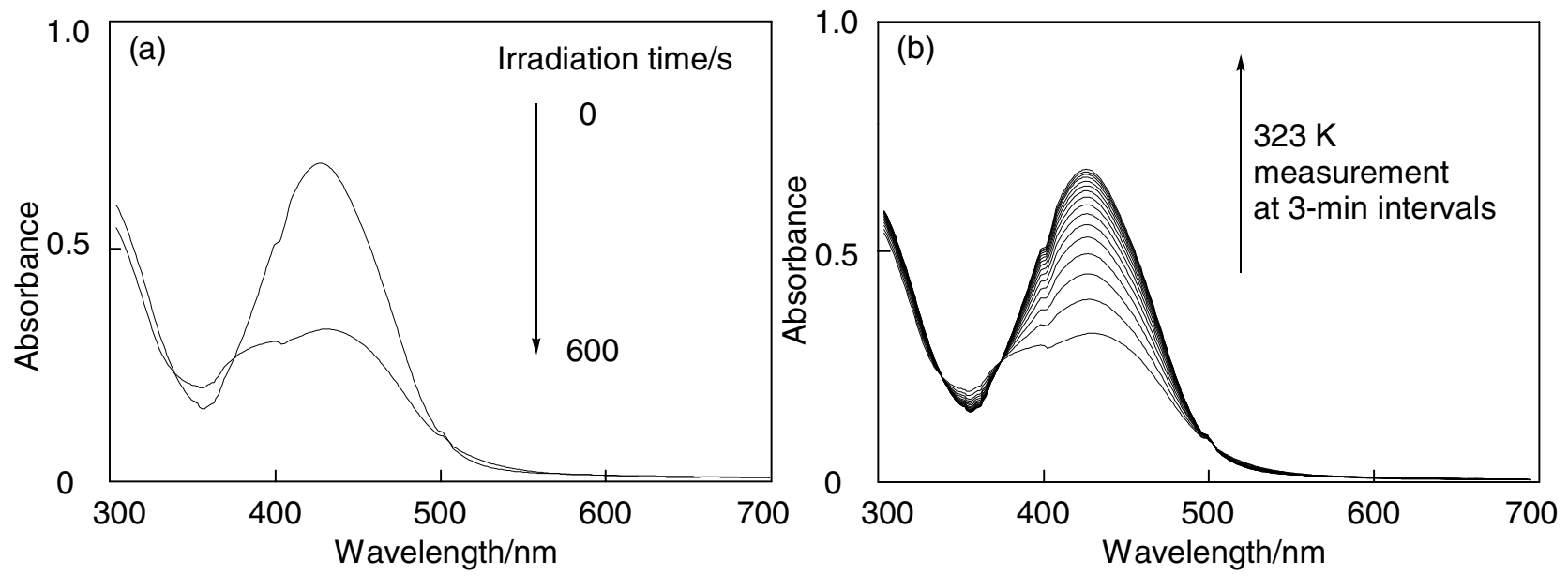

Figure S3. UV-vis spectra of trans-1 during (a) photoisomerization of the trans isomer by irradiation at $420 \mathrm{~nm}$ from a cut-off filterd xenon lamp at $288 \mathrm{~K}$ in toluene and (b) thermal isomerization of the cis/trans mixture at the photostationary state at $323 \mathrm{~K}$ in toluene.

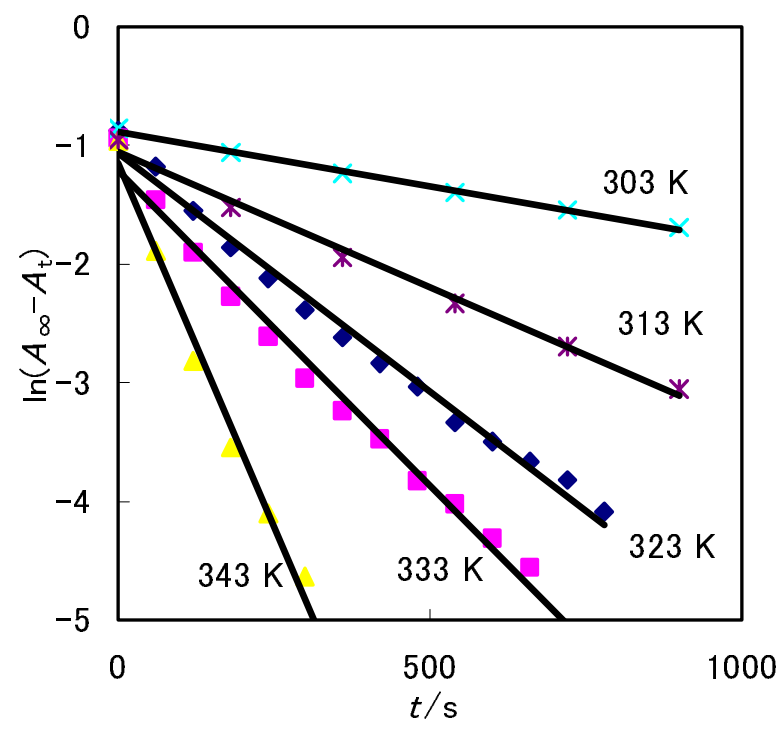

Figure S4. First-order plots of cis-trans isomerization of $\mathbf{1}$ in toluene.

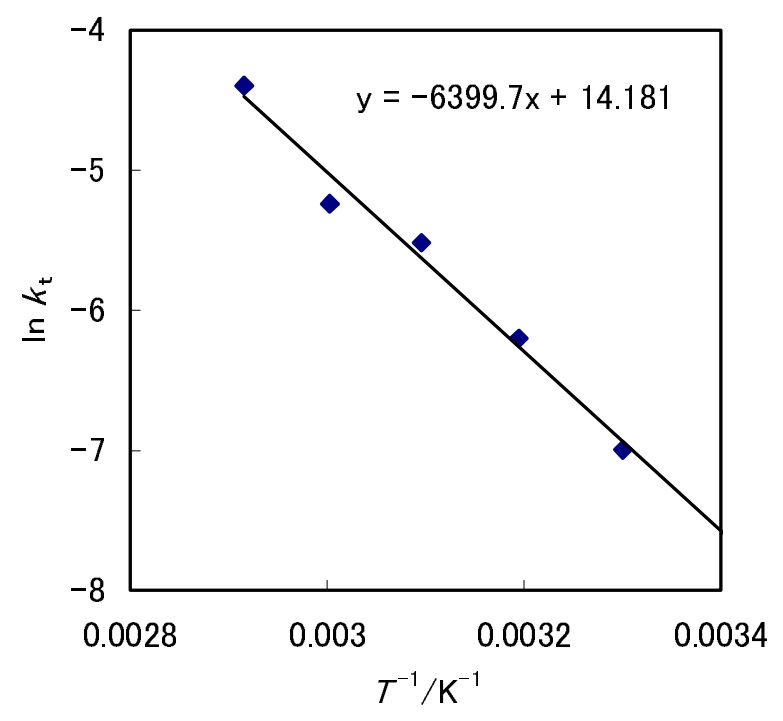

Figure S5. Arrehenius plots of the thermal isomerization of $\mathbf{1}$ in toluene. $E_{\mathrm{a}}=13 \mathrm{kcal} \mathrm{mol}^{-1}$. 